\section{Integralidad de las prácticas universitarias en el abordaje de conflictos socioambientales. Una experiencia docente desde la Universidad Nacional de Luján}

\author{
Alejandra Valverde \\ Consejo Nacional de Investigaciones \\ Científicas y Técnicas-Universidad Nacional \\ de Luján, Argentina. Asociación Argentino \\ Uruguaya de Economía Ecológica. \\ valverde.alejandra.5@gmail.com \\ (iD) orcid.org/0000-0002-0079-1338
}

Ambiente y extensión universitaria /
RECEPCIÓN: 29/04/21

ACEPTACIÓN FINAL: 25/06/21

\section{Resumen}

En el artículo se propone reflexionar acerca de la manera en la que desde nuestras asignaturas abordamos los conflictos socioambientales. Se busca interpelar además la forma en la que, desde nuestro rol docente, aportamos a la construcción de un pensamiento crítico orientado a la acción. La experiencia se desarrolla en el marco de dos asignaturas de la Licenciatura en Información Ambiental en la Universidad Nacional de Luján.

La integración de la teoría y la práctica implica trabajos en territorio, por lo que son necesarias estrategias pedagógicas que se articulen con la extensión universitaria. Es urgente una propuesta curricular para trabajar en los territorios en los que se expresan los conflictos por la apropiación, producción y distribución de bienes comunes, de modo de poder ampliar los espacios de diálogo con el estudiantado, los sujetos políticos y organizaciones sociales. A partir de dos casos de estudio se realiza un abordaje acerca del rol de la universidad en escenarios donde se desarrollan estos conflictos, en las dimensiones ambientales y territoriales de los mismos. La propuesta se enmarca en el ejercicio de la integralidad de las prácticas universitarias: docencia, investigación y extensión.

Palabras clave: conflictos socioambientales, extensión universitaria, integralidad.
Comprehensiveness of university practices in addressing socio-environmental conflicts. A teaching experience from the National University of Luján

\section{Abstract}

The article aims to reflect on the way in which we approach socio-environmental conflicts from our subjects. The proposal is to question the way in which, from our teaching role, we contribute to the construction of actionoriented critical thinking. The experience is developed within the framework of two subjects included in the major in Environmental Information, at the National University of Luján. The integration of theory and practice implies work in the territory, that's why pedagogical strategies that are combined with university extension are necessary. A curricular proposal to work in the territories in which conflicts are expressed because of the appropriation, production and distribution of common goods, in order to be able to expand the spaces for dialogue with the student body, political subjects and social organizations is urgent. Based on two case studies, an approach about the role of the university in scenarios where these conflicts are developed in their environmental and territorial dimensions is made. The proposal is framed in the exercise of the integrality of university practices: teaching, research and extension.

Keywords: socio-environmental conflicts, university extension, comprehensiveness.
Integralidade das práticas universitárias na abordagem de conflitos socioambientais. Uma experiência de ensino da Universidade Nacional de Luján

\section{Resumo}

O artigo se propõe refletir sobre a forma como abordamos os conflitos socioambientais a partir de nossas disciplinas. Também busca questionar a forma como, a partir de nosso papel docente, contribuímos para a construção de um pensamento crítico voltado para a ação. A experiência é desenvolvida no âmbito de duas disciplinas do Curso de Licenciatura em Informação Ambiental, da Universidade Nacional de Luján.

A integração da teoria e da prática implica um trabalho no território, por isso são necessárias estratégias pedagógicas que se articulem com a extensão universitária. É necessária uma proposta curricular de trabalhar nos territórios em que se expressam os conflitos pela apropriação, produção e distribuição dos bens comuns, para poder ampliar os espaços de diálogo com o corpo discente, os sujeitos políticos e as organizações sociais.

A partir de dois estudos de caso, aborda-se o papel da universidade nos cenários de desenvolvimento desses conflitos, em suas dimensões ambiental e territorial. A proposta se enquadra no exercício da integralidade das práticas universitárias: ensino, pesquisa e extensão.

Palavras-chave: conflitos socioambientais, extensão universitária, integralidade.

Para citación de este artículo: Valverde, A. (2021). Integralidad de las prácticas universitarias en el abordaje de conflictos socioambientales. Una experiencia docente desde la Universidad Nacional de Luján. +E: Revista de Extensión Universitaria, 11(14), e0012. doi: 10.14409/ extension.2021.14.Ene-Jun.e0012 


\section{Introducción}

En el presente artículo se propone, por un lado, sistematizar un recorrido de trabajo y aprendizaje en extensión universitaria que involucra docentes y estudiantes de la Universidad Nacional de Luján (UNLu, provincia de Buenos Aires); y por otro lado el anclaje, las limitaciones y los desafíos hacia la integralidad de las prácticas. El recorrido inicia en el año 2013 de la mano de un equipo extensionista constituido esencialmente por estudiantes y graduades universitaries $^{1}$ que en la actualidad -2021— se completa con docentes, investigadores y luchadores ambientales. Es por ello que en los apartados donde se describe la experiencia se tomó la decisión de escribir en primera persona del plural, ya que se trata de un proceso de aprendizaje colectivo, aunque la autoría de este artículo sea singular.

Las prácticas de extensión universitaria para la transformación social y el rol de la universidad en los conflictos socioambientales son temas de debate permanente en el equipo de trabajo. Es por ello que se entiende a la extensión como una puerta de entrada a los territorios donde se expresan dichos conflictos, y la integralidad de las prácticas universitarias supone considerarla como el motor para la integración de las tareas de docencia y de investigación. Puesto que es una herramienta para la transformación de la sociedad, no puede existir de forma aislada, sin conectarse con la investigación y la docencia.

La experiencia que aquí se desarrolla, como se mencionó, comienza en 2013, con un colectivo de estudiantes, graduades y militantes estudiantiles de la UNLu, del que formé parte como graduada de la Licenciatura en Información Ambiental. Los dos proyectos iniciales que dan lugar al trabajo actual se enmarcan en las convocatorias con financiamiento tanto de la Secretaría de Políticas Universitarias como de la UNLu. Las motivaciones que llevan a este equipo a trabajar un conflicto socioambiental en una provincia del noroeste argentino se instalan inicialmente en la solidaridad de clase ante los hechos de militarización ${ }^{2}$ y represión a vecines por defender el territorio del avance de proyectos de explotación minera a cielo abierto en la ciudad de Tinogasta (Catamarca). Asimismo, como forma de afianzar las redes y lazos solidarios tendidos a la luz del debate acerca del rechazo por parte de la comunidad universitaria de la UNLu de los fondos del Yacimientos Mineros de Agua de Dionisio (YMAD) debido a las consecuencias ambientales, sociales y económicas que tiene la actividad minera a cielo abierto, que se desarrolló en el año 2009. Compartimos el reclamo por la tierra y el agua, y por ello pusimos a disposición nuestro conocimiento ambiental en formación para acompañar su lucha. En tanto, el otro conflicto en el que comprometemos nuestra participación fue la inundación de la ciudad de Luján en 2012, lo que devino en la lucha por la conservación de humedales.

\footnotetext{
1) Nota de la editora: el cambio de la vocal "a" o de la "o" por la "e" en la terminación de algunos artículos, pronombres o sustantivos que denotan un sentido binario varón/mujer obedece a una intencionalidad política del uso de un lenguaje no sexista que está impulsada por algunas expresiones de movimientos feministas y colectivos de LGTB. $+\mathrm{E}$ marcará en itálica aquellas expresiones que se utilicen con el fin de respetar dicha intencionalidad siempre que no exista riesgo de anfibología.

2) En febrero de 2012, vecines y asambleístas fueron brutalmente reprimidos por la policía mientras realizaban cortes en la Ruta $N^{\circ} 60$. La modalidad era selectiva e informativa, con el objetivo de informar a los automovilistas e impedir el paso de camiones que abastecen a esos yacimientos. La policía provincial desalojó el piquete con balas de goma, perros y gases lacrimógenos.
} 
En la actualidad formo parte del equipo decente de dos asignaturas y soy investigadora y extensionista. Actividades que me permitieron articular el contacto con los sujetos sociales en conflicto e incorporar las problemáticas en la planificación curricular de las asignaturas Gestión Ambiental y Comercio Internacional y Ambiente. Se ubican en el cuarto y quinto cuatrimestre, respectivamente, del Plan de Estudios de la Licenciatura en Información Ambiental ${ }^{3}$ de la UNLu. La primera asignatura tiene entre sus contenidos mínimos ${ }^{4}$ temas como la gestión ambiental pública y privada y los modelos de gestión asociados, el rol de las ONG y de la sociedad en la gestión de los recursos naturales. La segunda asignatura contempla el rol de los recursos en los grandes intercambios internacionales, los mecanismos del comercio internacional que operan como distribuidores de riqueza y circuitos de comercialización, entre otros.

La matriz común entre ambas es el abordaje desde los conflictos ambientales en sus múltiples dimensiones y escalas y presta especial atención al rol de los diferentes actores y actoras en los procesos de producción, distribución, cambio y consumo de los bienes comunes. Ello entendiendo a los mismos como sistemas complejos que requieren un abordaje inter/ transdisciplinar para su resolución, orientado a la elaboración de políticas en ese sentido.

Se considera la docencia universitaria como una práctica que debe ser integradora y a la vez transformadora. El punto de partida es el Proyecto Institucional de la UNLu del año 2009, donde se plantea que la investigación, la docencia y la extensión son los tres pilares que comprenden la función social de la universidad. Se propone así, como una de sus principales misiones, la democratización del conocimiento y la construcción conjunta de saberes para la transformación social entre sujetos universitarios.

El desarrollo de estas experiencias apunta a la práctica de una extensión crítica, relacionada con la idea de integralidad, de modo de generar un vínculo bidireccional con la sociedad y sus instituciones, que fomente espacios de organización autónomos como forma de construir respuestas (y posibles soluciones) a los conflictos socioambientales. Es por ello que se trabaja esencialmente con los sujetos sociales que son parte de la resistencia al avance del capital en sus territorios, donde se dan aprendizajes colectivos en cuanto al vínculo sociedad naturaleza y en la búsqueda de soluciones y/o alternativas productivas a la minería a gran escala en un caso, y a la ocupación de humedales en el otro. Desde esta perspectiva se ha podido participar de la construcción de valoraciones y discursos en la defensa del agua y la tierra.

En el artículo se efectúa inicialmente una conceptualización compartida acerca de conflictos socioambientales para luego avanzar en una propuesta consensuada de abordaje metodológico de los mismos. Continúa con el desarrollo de la materialización conceptual en la experiencia de extensión y posteriormente describe ambos casos y la articulación de las prácticas universitarias en torno a ellos. Para finalizar, se presenta un modelo de universidad respecto del cual aspiramos a ser parte de su construcción y unas reflexiones a modo de conclusión.

\section{¿Por qué trabajar desde los conflictos socioambientales?}

Los conflictos socioambientales son conflictos sociales y políticos, ya que implican luchas de poder generalmente asimétricas y en torno a recursos naturales, denominados bienes co-

3) Ofrecida en la UNLu,

4) Resolución CS N 108/97. 
munes por las organizaciones socioambientales. Se plantean en términos de controversias derivadas de diversas formas de valorización de la naturaleza y de sus servicios ambientales (Wagner, 2010). Estos conflictos refieren a debates y disputas que enfrentan a diferentes actores institucionales e intersectoriales y se expresan territorialmente en distintas escalas ante las amenazas que vulneran la calidad de vida de las personas en la relación sociedadnaturaleza (Valverde, Camarero, Ordoñez; 2015).

En línea con lo expresado con el Colectivo de investigadores e investigadoras ${ }^{5}$ (2013), no consideramos el conflicto como un episodio patológico que es necesario evitar, prevenir o desactivar lo más rápidamente posible, buscando una solución consensuada, negociada y aceptada por todos los participantes de la discusión. Rescatamos las perspectivas que lo entienden como un escenario complejo donde se expresan los discursos a través de la toma de palabra y las prácticas sociales. Estos se insertan en contextos geopolíticos específicos expresados territorialmente que develan una conexión entre las tendencias económicas que orientan la producción capitalista y la destrucción del medio ambiente, puesto que una no es sin la otra. La destrucción ya no se visualiza en términos del entorno sino que es transversal, en los aspectos sociales, culturales, políticos.

Merlinsky afirma que los conflictos socioambientales:

"Representan focos de carácter político que generan tensiones en las formas de apropiación, producción, distribución y gestión de los recursos naturales en cada comunidad o región. Ponen en cuestión las relaciones de poder que facilitan el acceso a esos recursos, que implican la toma de decisiones sobre su utilización por parte de algunos actores y la exclusión de su disponibilidad para otros actores. Se trata de situaciones de tensión, oposición y/o disputa en la que no solo están en juego los impactos ambientales". (2014, p. 40)

Lo más relevante de este tipo de perspectivas es asumir la complejidad sistémica que involucran los conflictos, especialmente el rol de les actores sociales con capacidad de definirse a sí mismos y generar una acción pública colectiva, y la trama de las relaciones de poder que se expresan en diferentes racionalidades, valoraciones e intereses.

Este tipo de conflictos conlleva, además, riesgos ambientales en los que los hechos son inciertos, las valoraciones están en disputa, lo que se pone en juego es alto, y las decisiones son urgentes (Funtowicz, Ravetz, 1993). Retomamos la perspectiva de complejidad y emergencia de los riesgos ambientales y la necesidad de tomar decisiones urgentes bajo la figura del consenso público y la participación ciudadana. No partimos de una teoría general asociada a una disciplina particular; por el contrario, apuntamos a la resolución de problemas específicos que generan conflictos socioambientales desde una perspectiva de ciencia posnormal para la acción. En ese sentido, García (2011) abona a la misma desde un enfoque sistémico y habilita a la elaboración de una metodología de estudio novedosa y específica para el abordaje de los problemas ambientales partiendo del conflicto en tanto sistema complejo.

Esta conceptualización propone lograr una síntesis integradora de los elementos del sistema complejo acudiendo a la interdisciplinariedad como condición fundamental para el abordaje de los mismos. Tendemos a la interdisciplina ante la necesidad de reformular prác- 
ticas de la ciencia para el análisis de los problemas ambientales complejos, para la interpretación de las interacciones que se dan en dicho sistema entre los procesos que determinan su funcionamiento (García, 2011). El estudio de las problemáticas ambientales emergentes en el proceso de toma de decisiones pone en juego mayores costos que en la ciencia aplicada y/o en la consultoría profesional. Este enfoque implica tener en cuenta la incertidumbre inherente a los hechos y a los valores que están en conflicto y asimismo requiere de decisiones urgentes, consenso público y participación ciudadana (Funtowicz, 1993).

\section{Propuesta metodológica para la intervención en los conflictos ambientales desde la extensión universitaria}

La propuesta de trabajo que aquí se desarrolla es construida de manera colectiva, tanto teórica como empíricamente. Toma como base metodológica herramientas cualitativas de investigación que involucran la investigación-acción y la extensión crítica como práctica para el abordaje de la complejidad desde un enfoque sistémico.

La práctica de la extensión crítica contempla la aplicación del conocimiento científico y los saberes situados en la gestión de conflictos sociales asociados a lo ambiental-territorial y apunta a la inclusión de las voces de todes les actores que se involucran, teniendo como planos de acción los espacios de la academia y los laborales, las prácticas de militancia y el trabajo social.

El objetivo es lograr una interpretación sistémica de los conflictos socioambientales, de manera de llegar a un diagnóstico integrado y participativo que provea las bases para promover acciones concretas y políticas públicas que permitan influir el sistema ambiental. Apostamos así a la co-construcción de un conocimiento que posibilite la articulación entre distintos campos disciplinares y saberes situados para elaborar una metodología interdisciplinaria adecuada para el abordaje de los conflictos socioambientales.

La propuesta parte de considerar les actores y las relaciones sociales con la finalidad de construir diagnósticos participativos que sirvan como insumo para la elaboración de propuestas para la acción. Estas involucran los siguientes enfoques:

1. Enfoque desde les actores. Análisis institucional como forma de abordaje y caracterización de les mismes y sus relaciones sociales. Análisis del posicionamiento en el campo social en cuanto a discursos, prácticas, participación en decisiones y relaciones con otros, historicidad y trayectorias de vida, intereses y demandas, valoración del ambiente, etcétera.

2. Enfoque desde las relaciones de poder. El espacio social se analiza como un campo construido a partir de relaciones de poder que se ejercen entre quienes se disputan el uso de un recurso determinado en un lugar determinado. Entendemos el espacio social tanto territorial como institucional, comunicacional y de influencia. En ese marco conceptual es necesario realizar un análisis que nos permita definir los intereses e identificar la trama de relaciones de poder. Observamos así distintos campos donde se ejerce el poder, que es construido en torno a les actores que intervienen en un conflicto (Valverde Camarero, Ordoñez, 2015). Se analizan aspectos tales como:

a) Niveles y escalas de impacto y la capacidad de organización en torno al problema ambiental. 
b) Apropiación del discurso ambiental y percepción de cuál es el conflicto, sus responsables y posibles soluciones.

c) Relaciones entre alianzas, influencias y transformaciones.

d) Contexto institucional y normativo para el accionar en la arena política.

3. Diagnóstico participativo. Para poder definir herramientas para la acción se parte de la necesidad de hacer un diagnóstico que identifique elementos tales como problemas de comunicación, diferentes interpretaciones y visiones del conflicto y las categorías discursivas, asimetrías de poder, distintos saberes y racionalidades. Cualquier proceso de aprendizaje y diagnóstico participativo debe incluir la intervención de todes les involucrades en el conflicto.

4. Propuestas para la acción. Se construye colectivamente una serie de propuestas que pueden acercar a intervenciones concretas, de manera que, al mismo tiempo que se realizan los esfuerzos para interpretar las relaciones que dan cuenta del conflicto en el sistema ambiental, se pueda intervenir en la política pública con y desde les actores, a fin de encontrar soluciones en el mediano y largo plazo. Todas estas propuestas están alimentadas de saberes y aprendizajes colectivos y refieren a:

a) Construir roles de facilitadores/mediadores sociales.

b) Co-construir conocimiento.

c) Generar espacios de organización a partir de la interpretación de los conflictos como oportunidad.

\section{De la propuesta metodológica a la experiencia en extensión universitaria}

La necesidad de incorporar la extensión a las asignaturas no surge al momento de debatir los contenidos sino como acumulación colectiva puesta a disposición para trabajar con les integrantes del equipo docente. ${ }^{6}$ Dicho equipo está constituido por docentes que venimos de varias trayectorias académicas y experiencias organizativas, una de ellas es la que le da cuerpo a la propuesta de inclusión de los conflictos socioambientales en la currícula. La misma emerge de la participación en un proyecto de extensión previo, desarrollado en el seno de la UNLu e impulsado por el Frente Universitario de Luján, que abrió las puertas para trabajar el conflicto de manera conjunta entre universidad y actores territoriales.

Las convocatorias institucionalizadas que contaron con financiamiento para extensión no solo nos han permitido empezar a andar el camino de la integralidad de las prácticas universitarias sino que nos han dado la posibilidad de integrar nuestras luchas territoriales por una sociedad in injusticias, de modo de hacer transitar por la misma senda al conocimiento universitario y al conocimiento teórico y técnico producido en los territorios, y construir colectivamente otros, con base en las ciencias y en los saberes populares, aprendidos en el calor de la lucha por un ambiente sano y por elevar la calidad de vida de la población.

6) Se trata de una acumulación en el marco del Colectivo del que participo, Frente Universitario de Luján, que toma contacto con el territorio y sus actores inicialmente en el apoyo de la lucha antiminera. Esas relaciones se plasman en un proyecto de extensión que más adelante es tomado para ser incorporado en la asignatura Comercio Internacional y Ambiente. 
A continuación se realiza una descripción del camino que se ha recorrido hasta el momento en el marco de las experiencias de trabajo entre territorio y universidad. Cada una de ellas inició como proyecto de voluntariado universitario y se fue transformando en eje estructurante de contenidos curriculares y posteriores iniciativas de extensión para dar continuidad al trabajo iniciando.

\section{En defensa del agua, el cerro y la vida en la provincia de Catamarca}

El primer recorrido referencia el trabajo con la Asamblea de Tinogasta Darío Moreno en el marco del conflicto socioambiental debido a la resistencia al avance de la explotación minera a cielo abierto en el Valle del río Abaucán (Tinogasta, Catamarca). La Figura 1 esquematiza esa trayectoria.

Figura 1. Síntesis de la experiencia "En defensa del agua, el cerro y la vida en la provincia de Catamarca"

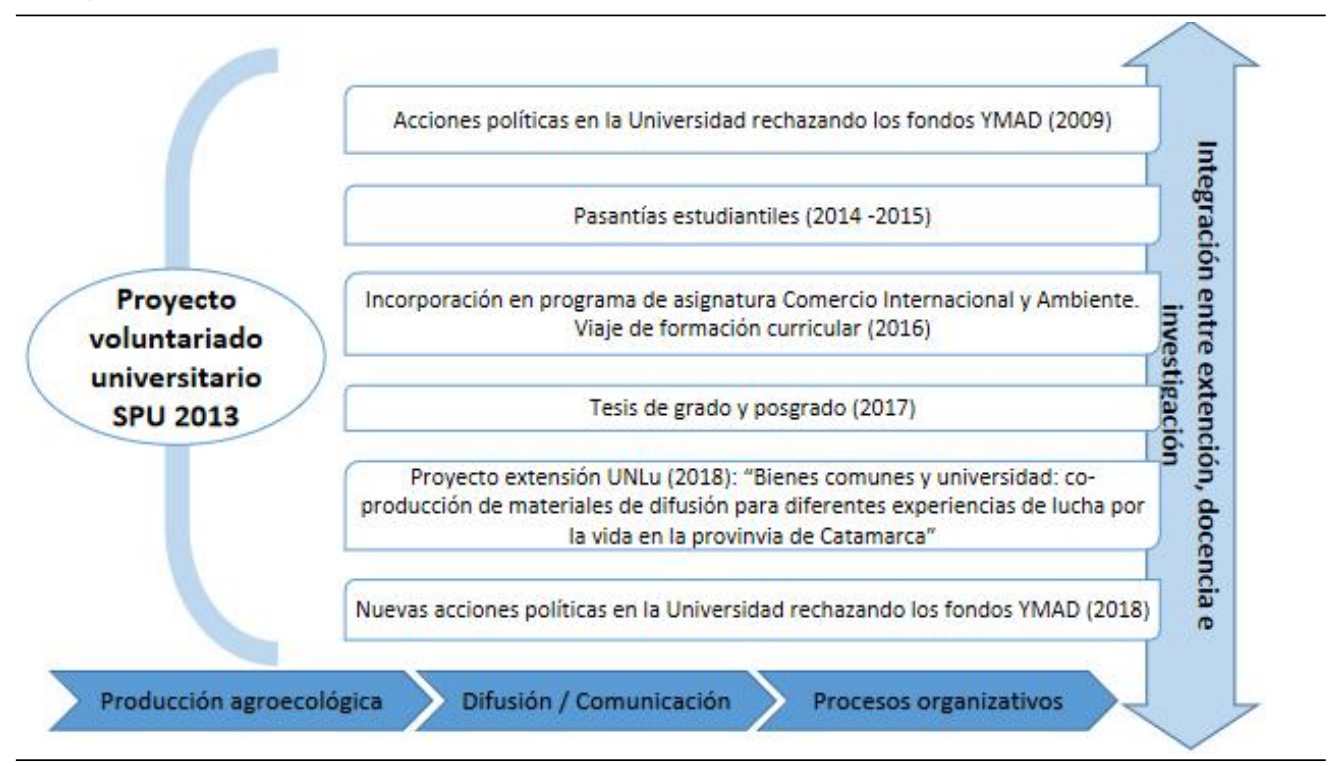

Fuente: elaboración propia, 2021.

\section{El contexto}

La década del 90 significó un fuerte clivaje para la reconfiguración de las economías regionales. La implementación de las medidas neoliberales promovió la concentración productiva y financiera y la eliminación de organismos estatales de promoción, lo que llevó a la paulatina desaparición de pequeños y medianos productores, a la disposición de las economías regionales hacia la exportación y a una desarticulación del entramado social que sostenía este tipo de economías. Los altos índices de desempleo, la gran dependencia de su población del empleo público y de los planes sociales, y el crecimiento del trabajo informal se profundizaron en Catamarca. Los índices de pobreza e indigencia, la pauperización de las condiciones de vida y la ausencia de derechos básicos, entre ellos el derecho a una alimentación sana, nutritiva, adecuada en cantidad y culturalmente aceptable, generaron alarmas. 
Respecto de estos procesos de apropiación y avance sobre los territorios, la universidad pública no es ajena, el capital busca legitimación y consenso. En medio de la conflictividad social ocasionada por el avance de la minería en nuestra cordillera, el Estado nacional y los capitales privados despliegan estrategias en ese sentido. YMAD es una sociedad compuesta entre la Universidad Nacional de Tucumán, el Estado catamarqueño y el Estado nacional ${ }^{7}$ que, a mediante la Ley Nacional 14.771, sancionada en 1958, tiene la obligación de distribuir utilidades entre las universidades nacionales. Así, en 2009 comenzó a implementarse la distribución de ese dinero y se abrió el debate, no solo con relación al financiamiento por parte del sector privado a la universidad pública, sino también acerca de las implicancias de que ese dinero viniera de una actividad fuertemente cuestionada por sus consecuencias ambientales. Dicha situación movilizó las distintas fuerzas estudiantiles y docentes y a la comunidad universitaria en general y se inició un proceso de discusión sobre el presupuesto y la construcción de conocimientos y, en especial, en cuanto al rol que jugaría la universidad en ese conflicto. Luego de varios meses de agitación y actividades públicas, en diciembre de 2009 se dio la discusión en el Honorable Consejo Superior a raíz de un pedido del estudiantado por rechazar los fondos provenientes de YMAD. Finalmente, por resolución ${ }^{8}$ de este órgano de cogobierno, se repudiaron públicamente los daños sociales, ambientales, económicos y políticos ocasionados por el modo extractivo a cielo abierto y se rechazaron los fondos provenientes de la aplicación de la Ley 14.771 y todo fondo proveniente de las regalías de la actividad minera a cielo abierto. De este modo la UNLu se convirtió en una de las primeras universidades nacionales que rechazó dichos fondos.

En este contexto y puestos el tema y el conflicto en las agendas políticas de los gobiernos por parte de las organizaciones sociales y estudiantiles, fue preciso pensar de qué manera la universidad podía aportar a la mejora de la calidad de vida. En medio de estos debates, se dieron procesos cargados de violencia institucional por parte del Estado y los capitales mineros, que reprimieron fuertemente las acciones públicas de les vecines organizades en la provincia de Catamarca (Andalgalá y Tinogasta), quienes se habían propuesto informar y concientizar a la población en general. Rápidamente las imágenes de represión y conflictividad llegaron a los medios de comunicación nacionales, lo que generó un gran apoyo a la lucha contra la megaminería a cielo abierto.

Un grupo de estudiantes y docentes vieron entonces que era imperioso apoyar y acompañar la lucha con la herramienta que se tenía en ese momento, un conocimiento ambiental en formación. Así se pusieron en marcha estrategias que dieran cuenta de la función social de la universidad y su compromiso con la sociedad. Se generó un vínculo con la Asamblea de Tinogasta Darío Moreno, desde donde se problematizó la cuestión del acceso y la calidad del agua, de la alimentación y de la autodeterminación de los pueblos. Este proceso de debate y aprendizaje fue el insumo para la experiencia que aquí se describe.

También se planteó, en conjunto con la Asamblea, un proyecto de extensión para promover la realización de una huerta agroecológica comunitaria en pequeña escala en Tinogasta (Catamarca), de modo de fomentar la participación de la comunidad y ejercer un efecto

7) Tiene bajo su concesión el yacimiento Bajo La Alumbrera, explotado desde hace quince años por el consorcio suizo-canadiense Xstrata (50\%), Goldcorp (37,5\%) y Yamana Gold (12,5\%), y extrae oro y cobre. 8) REDHCSLUJ:502/09. 
multiplicador en lo que respecta a las producciones con enfoque agroecológico. Algo que al mismo tiempo estimulara el desarrollo de economías regionales a partir de la recuperación de saberes prácticos asociados al trabajo sustentable con la tierra, la creación de empleo digno, la alimentación saludable y la consolidación de un ambiente sano y sustentable, contribuyendo al mejoramiento de la calidad de vida.

En el año 2013 se aprobó el proyecto "Economías regionales, sustentabilidad ambiental e integración territorial: la construcción de una huerta agroecológica comunitaria en Tinogasta, provincia de Catamarca". Esta propuesta de extensión se elaboró a partir de la relación y el trabajo político territorial entre el Frente Universitario de Luján y la Asamblea de Tinogasta Darío Moreno, en apoyo a la lucha contra la megaminería a cielo abierto y las consecuencias ambientales de la actividad. Fue presentado en la $15^{\circ}$ Convocatoria de Proyectos de Extensión Universitaria y Vinculación Comunitaria (2013) de la Secretaría de Políticas Universitarias y forma parte de los proyectos de extensión del Programa de investigación Redes Epistémicas Ambientales, del Departamento de Ciencias Sociales de la UNLu.

En el marco del mismo se han desarrollado herramientas teórico-prácticas asociadas a la perspectiva del cooperativismo, la economía social y solidaria, a partir de la problematización del modelo extractivo. Se estimuló la práctica agroecológica como forma alternativa de producción, apoyándose en la educación ambiental como herramienta fundamental del desarrollo social y económico de la región. Además se generaron líneas de trabajo en cuanto a la forma de comunicar posiciones y resultados por parte de la comunidad local, lo que promovió la instalación de una radio comunitaria. Se crearon espacios de formación con quienes participaban directamente del trabajo de la huerta, la cooperativa y la radio con la comunidad. En este proceso, la Universidad (el equipo extensionista) participó en todas las instancias, asesorando, aprendiendo nuevos saberes y constituyendo espacios de construcción colectiva de conocimiento.

Las actividades planificadas en el proyecto contemplaban nueve meses de trabajo en los que participaron al menos 20 estudiantes de distintas carreras de la UNLu ${ }^{9}$ de manera estable, y alrededor de 10 en instancias puntuales. Todas fueron planificadas entre estudiantes, graduades y asambleístas a medida que se iba dando forma a la propuesta. Algunas de las actividades fueron las siguientes:

- Talleres de agroecología, salud y ambiente, microemprendimientos, radio comunitaria y mapeo colectivo de problemáticas ambientales.

- Instalación y desarrollo de la huerta agroecológica comunitaria.

- Pasantías estudiantiles con recorrido por la región para el reconocimiento de las actividades productivas.

- Entrevistas a productores, productoras y autoridades políticas de la zona.

- Actividades de difusión política en la Universidad. Por RESHCS-LUJ 502/09 la UNLu plasmó un proceso de debate a nivel de comunidad universitaria con relación a los efectos de la actividad minera a cielo abierto. Es por ello que el equipo de trabajo decidió dar 
continuidad a las decisiones y resoluciones allí planteadas, difundir por distintos medios el trabajo desarrollado en el marco del proyecto y ampliar la convocatoria a extensionistas.

- Presentación de avances en eventos académicos y de extensión.

Finalizados los plazos administrativos del proyecto hacia mediados del año 2015, se impuso la necesidad de continuar el trabajo territorial. De este modo, de la mano del movimiento estudiantil de la Universidad se impulsaron pasantías estudiantiles con el fin de abrir la convocatoria a más estudiantes con interés en la temática. De las mismas participaron 10 estudiantes de distintas carreras y fueron financiadas de manera autónoma por el Frente Universitario de Luján con el apoyo de centros de estudiantes. ${ }^{10}$ Sus principales objetivos fueron:

- Promover el fortalecimiento de los lazos comunitarios y solidarios entre la comunidad y la

Universidad, creando instancias de conocimiento enriquecidas por la unión de los saberes populares y el conocimiento científico, más allá del proyecto de extensión finalizado.

- Motivar instancias organizativas estudiantiles.

- Incentivar la investigación científica, el trabajo interdisciplinario y colectivo, el desarrollo de actividades de extensión universitaria y la incorporación de las temáticas en asignaturas.

- Problematizar críticamente el rol de la Universidad en conflictos socioambientales generados a partir de la explotación minera a cielo abierto.

- Fomentar el debate amplio y democrático sobre las problemáticas territoriales/ambientales que caracterizan las distintas realidades locales, nacionales e internacionales al interior de la Universidad.

Desde la multiplicidad de disciplinas y recorridos de formación, estas pasantías desarrollaron actividades de formación a partir de talleres y charlas con los diferentes grupos de trabajo locales, el reconocimiento de los emprendimientos productivos de la zona, recorrido por Fiambalá, Copacabana, Aimogasta, Andalgalá y Saujil, con el objetivo de definir de forma colectiva las estrategias productivas que caracterizan la economía regional de Tinogasta, y formarse en el trabajo y sostenimiento de un medio de producción comunitario, alternativo, popular y sin necesidad de megaminería (actividad "productiva" que a la fecha no cuenta con licencia social).

Las pasantías permitieron al equipo dar continuidad al trabajo iniciado en el proyecto y plantear algunas líneas de trabajo futuro. Durante 2016 se intentó replicar la experiencia pero sin éxito ya que la dificultad más grande fue el financiamiento, por lo que el equipo se dedicó ese año a sistematizar el trabajo realizado y los aprendizajes colectivos en publicaciones y ponencias para eventos académicos.

Los procesos de formación comunitarios y la acción política en distintos ámbitos generadas a partir de la extensión universitaria tuvieron una gran influencia en nuestra vida profesional y política. Es por ello que lo acumulado colectivamente a partir del proyecto inicial siguió un curso propio y les integrantes del equipo tomaron el tema para investigación en tesis de grado y posgrado y, en mi caso, se introdujo en los contenidos curriculares de una asignatura.

10) Centro de Estudiantes de la Licenciatura en Información Ambiental, Centro de Estudiantes del Profesorado en Geografía y Centro de Estudiantes de Ingeniería Industrial. 
De un proyecto de voluntariado a la currícula

Se inició un proceso de debate al interior del equipo docente del que formo parte, favorecido por los distintos recorridos teóricos y políticos de cada integrante docente, que se cristalizó en una propuesta actualizada del Programa de la asignatura Comercio Internacional y Ambiente. ${ }^{11}$ Esta se aborda desde la Economía Ecológica y Ecología Política a partir de la integralidad de las prácticas universitarias en torno a los conflictos socioambientales. Se incorporaron nuevas estrategias pedagógicas y en especial la extensión universitaria como práctica integradora. No solo con la docencia y la investigación, sino también como práctica para poder analizar los conflictos socioambientales de manera integral. Se seleccionó como caso testigo para analizar las implicancias ambientales del comercio internacional la producción megaminera a cielo abierto en la provincia de Catamarca (Argentina). Es una actividad que en parte puede generar ventajas comparativas y aumento de reservas monetarias, pero que genera extractivismo de bienes comunes, acumulación por desposesión, desigual acceso a los territorios, pobreza y contaminación ambiental.

En el trabajo con conflictos socioambientales, el despliegue de marcos conceptuales y teóricos no es suficiente. Se entiende a la docencia universitaria como una práctica que debe ser integradora y a la vez transformadora, por lo que la forma de trabajo intenta comprometer a les estudiantes de las diferentes cohortes a que sigan realizando un aporte a la lucha socioambiental antes mencionada.

Vinculando actores sociales en una problemática común, sobre la base del trabajo y acumulación de aprendizajes a partir de la extensión, se propuso como estrategia pedagógica un viaje curricular para la asignatura, de manera de integrar la teoría y la práctica con trabajos en territorio. Se procuró aproximarse al conocimiento crítico de los procesos abordados en el Programa de la asignatura. El viaje tuvo una duración de seis días y se trabajó durante el cuatrimestre la hoja de ruta anterior y la posterior sistematización.

Síntesis de la experiencia docente (2017-1019)

Se buscó un acercamiento entre teoría y práctica en el territorio que se materializó con la incorporación del viaje curricular a la provincia de Catamarca en el marco de la asignatura. En el mismo se propuso problematizar las relaciones entre sociedad y naturaleza a partir de un estudio de caso territorializado: la producción minera a cielo abierto y su vínculo con el comercio internacional y el ambiente. Se desarrolló una serie de actividades que, además de suponer un alto compromiso de estudiantes y docentes, requirieron de la participación de les actores locales implicados en el conflicto socioambiental abordado.

El viaje curricular articuló tres ejes transversales contemplados desde la asignatura: los estilos de desarrollo implementados en la región de América Latina; los conflictos socioambientales originados por los diferentes modelos de acumulación de capital; y los debates sobre el desarrollo sustentable y alternativas al desarrollo. La bibliografía de base es la que se presenta en el Programa de la asignatura y al mismo tiempo se requirió de búsqueda bi-

11) Asignatura que corresponde al quinto cuatrimestre del Plan de Estudios de la Licenciatura en Información Ambiental (UNLu). Pertenece a un grupo de cuatro asignaturas de la carrera que se enmarca en la división Economía del Departamento de Ciencias Sociales. A partir de los contenidos mínimos (RCS 108/97) retoma los análisis vigentes desde mediados del siglo XX y encara aspectos económicos de los conflictos socioambientales. 
bliográfica específica por parte de les estudiantes, en especial para la resolución del trabajo práctico final integrador. El viaje cumplió una función formativa y de aprendizaje en el diálogo e intercambio con les actores sociales que tomó fuerza en la nueva propuesta de trabajo extensionista que se describe más adelante. Se presenta en el siguiente cuadro una síntesis de las actividades que se realizaron y las articulaciones en cada caso.

Cuadro 1. Actividades del viaje curricular y procesos compartidos

\begin{tabular}{|c|c|c|c|}
\hline Territorio & Actividad & Actores sociales & Resultados/ procesos \\
\hline \multirow[t]{2}{*}{$\begin{array}{l}\text { San Fernando } \\
\text { del Valle de } \\
\text { Catamarca. } \\
\text { UNCa }\end{array}$} & $\begin{array}{l}\text { Taller: Formas de desa- } \\
\text { rrollo y políticas ambien- } \\
\text { tales. } \\
\text { Ejes: } \\
\text { - Estilos de Desarrollo, } \\
\text { - Rol del Estado y } \\
\text { - conflictos socioam- } \\
\text { bientales. }\end{array}$ & $\begin{array}{l}\text { Organizado en conjunto con la } \\
\text { cátedra Problemáticas Culturales } \\
\text { del Mundo Contemporáneo, del } \\
\text { Dpto. de Ciencias de la Educación, } \\
\text { Facultad de Humanidades, UNCa. } \\
\text { Participan estudiantes y docentes } \\
\text { de ambas asignaturas de UNCa y de } \\
\text { UNLu, y se invita a integrantes de las } \\
\text { asambleas ambientales locales. }\end{array}$ & $\begin{array}{l}\text { Se problematiza acerca de la relación sociedad-na- } \\
\text { turaleza y se amplía la base conceptual desde una } \\
\text { articulación entre antropología, economía, socio- } \\
\text { logía y ciencias ambientales. Les estudiantes de } \\
\text { las dos universidades tienen la posibilidad de inter- } \\
\text { cambiar vivencias y experiencias y de sensibilizarse } \\
\text { con los conflictos de ambos territorios. }\end{array}$ \\
\hline & $\begin{array}{l}\text { Recorrida por la ciudad: } \\
\text { entrevista a les dife- } \\
\text { rentes actores sociales } \\
\text { con cuestionario guía } \\
\text { elaborado para cada uno } \\
\text { de los lugares a visitar } \\
\text { (como segunda parte del } \\
\text { taller). }\end{array}$ & $\begin{array}{l}\text { Oficina de Minería Alumbrera, } \\
\text { YMAD, CAMYEN SE, Dirección } \\
\text { Provincial de Gestión Ambiental, } \\
\text { Secretaría de Minería, Secretaría } \\
\text { Académica de la UNCa, Secreta- } \\
\text { ría de Ciencia y Tecnología de la } \\
\text { UNCA, Centro de Estudiantes de las } \\
\text { Facultades de la UNCa. }\end{array}$ & $\begin{array}{l}\text { Se reconocen las "huellas" de la minería en la ciu- } \\
\text { dad a través de la identificación de murales, pinta- } \\
\text { das, carteles, etcétera. } \\
\text { Se analizan las entrevistas y se realiza una puesta } \\
\text { en común que permite identificar discursos (am- } \\
\text { bientales, productivistas, vivenciales). Les estudian- } \\
\text { tes tienen una "entrada" a la problemática desde } \\
\text { sus percepciones. }\end{array}$ \\
\hline \multirow[t]{4}{*}{ Andalgalá } & $\begin{array}{l}\text { Almuerzo de bienvenida } \\
\text { y entrevistas. }\end{array}$ & $\begin{array}{l}\text { Integrantes de la Asamblea } \\
\text { El Algarrobo. }\end{array}$ & $\begin{array}{l}\text { Se reconoce a les actores que están en territorio, su } \\
\text { historia de lucha y su cosmovisión en la defensa de } \\
\text { "su lugar". Se propicia el diálogo con sujetos que } \\
\text { hasta el momento no conocían. }\end{array}$ \\
\hline & $\begin{array}{l}\text { Vista a Feria local y } \\
\text { salida en vivo en la radio } \\
\text { El Algarrobo. }\end{array}$ & $\begin{array}{l}\text { Productores y productoras locales. } \\
\text { Periodistas/asambleístas locales. }\end{array}$ & \multirow{2}{*}{$\begin{array}{l}\text { En el diálogo con productores, in situ, se conocen } \\
\text { las prácticas orgánicas y agroecológicas para el } \\
\text { trabajo productivo. Se identifican las diferencias } \\
\text { entre ambas y se aprenden técnicas agroecológicas } \\
\text { y formas de comercialización. Se debate acerca de } \\
\text { la necesidad de producir la tierra sin megaminería. }\end{array}$} \\
\hline & $\begin{array}{l}\text { Taller en finca de transi- } \\
\text { ción agroecológica en la } \\
\text { localidad El Potrero. }\end{array}$ & $\begin{array}{l}\text { Productores y productoras locales y } \\
\text { técnica de la Subsecretaría de Agri- } \\
\text { cultura Familiar. }\end{array}$ & \\
\hline & $\begin{array}{l}\text { Participación en la Cami- } \\
\text { nata por la vida. }\end{array}$ & $\begin{array}{l}\text { Asamblea El Algarrobo, vecines de } \\
\text { Andalgalá. }\end{array}$ & $\begin{array}{l}\text { Al participar en la acción política local se tienden } \\
\text { lazos solidarios con la lucha. }\end{array}$ \\
\hline Tinogasta & $\begin{array}{l}\text { Taller: Conociendo alter- } \\
\text { nativas en Agroecología } \\
\text { y circuitos de comercia- } \\
\text { lización. } \\
\text { Taller realizado in situ, } \\
\text { en fincas de producción } \\
\text { agoecológicas. }\end{array}$ & $\begin{array}{l}\text { Productores y productoras locales y } \\
\text { técnica del INTA. }\end{array}$ & $\begin{array}{l}\text { En el diálogo con productores, in situ, se conocen } \\
\text { las prácticas orgánicas y agroecológicas para el } \\
\text { trabajo productivo. Se identifican las diferencias } \\
\text { entre ambas y se aprenden técnicas agroecológicas } \\
\text { y formas de comercialización. Se debate acerca de } \\
\text { la necesidad de producir la tierra sin megaminería. }\end{array}$ \\
\hline \multirow[t]{2}{*}{ Fiambalá } & $\begin{array}{l}\text { Desayuno de bienvenida } \\
\text { y entrevistas. }\end{array}$ & Asamblea Fiambalá despierta. & $\begin{array}{l}\text { Se reconoce a les actores que están en territorio, } \\
\text { su historia de lucha y su cosmovisión en la defen- } \\
\text { sa de "su lugar". Se propicia el diálogo con sujetos } \\
\text { que hasta el momento no conocían. }\end{array}$ \\
\hline & $\begin{array}{l}\text { Recorrido en escuelas y } \\
\text { visita a la radio local para } \\
\text { contar la experiencia. }\end{array}$ & $\begin{array}{l}\text { Estudiantes y docentes de nivel me- } \\
\text { dio de la ciudad de Fiambalá. Perio- } \\
\text { dista radio local. }\end{array}$ & $\begin{array}{l}\text { Luego de varios días de intercambios y aprendiza- } \\
\text { jes, les estudiantes pueden contar la experiencia, } \\
\text { sensaciones y vivencias, tanto en la radio como en } \\
\text { las escuelas que les abren las puertas para ha- } \\
\text { cerlo, al mismo tiempo que se sigue aprendiendo } \\
\text { colectivamente. }\end{array}$ \\
\hline
\end{tabular}

Fuente: elaboración propia (Valverde, 2020). 
El trabajo necesitó de la preparación previa de las actividades, ya que les estudiantes en grupos de trabajo tomaron la responsabilidad y compromiso para la realización y desarrollo de cada jornada en cada uno de los territorios. Las planificaciones y hoja de ruta se elaboraron previamente en el aula. Durante las actividades in situ, los diferentes grupos tenían la tarea de llevarlas adelante y sistematizarlas. Por último, de regreso a la dinámica áulica, debían entregar un informe de todo lo trabajado donde cumplieran con una serie de consignas dadas por el equipo docente. El trabajo integrador se entregaba por escrito y se defendía en forma de coloquio junto con todo el curso. ${ }^{12}$

A lo largo de la experiencia es posible indagar en territorio acerca de los procesos productivos que involucra la actividad de la megaminería a cielo abierto y sus consecuencias directas en el ambiente, la sociedad y sus condiciones de vida. Es de conocimiento público que, además de los cuestionados beneficios económicos que produce la actividad, registra conflictos a raíz de la contaminación y la escasez de agua, visibilizados a partir de las protestas y acciones públicas de la comunidad.

Los casos concretos que se tomaron para el análisis son los procesos protagonizados por los departamentos de Andalgalá, Tinogasta y Fiambalá. Ciudades con megaemprendimientos mineros en sus cercanías y con una historia de lucha por el territorio y búsqueda de alternativas al modelo extractivo de desarrollo planteado hasta el momento. Asimismo, se observaron las posibilidades concretas de actividades productivas alternativas al discurso del pensamiento único, que plantea como opción viable exclusiva la instalación de megaemprendimientos de extracción y exportación de recursos. Estas alternativas, que incluyen producciones agroecológicas, permiten contrarrestar los efectos de la actividad megaminera, tales como los desplazamientos de campesinos y productores locales y la pérdida del conocimiento local.

La experiencia de trabajar en los territorios habilita a les estudiantes tomar nota de los discursos y demandas de las comunidades locales, que son atravesadas por la problemática. A partir de allí, luego de materializado el viaje curricular, pusieron en marcha distintos dispositivos en el marco de su "quehacer" como estudiantes, desde la elaboración de notas periodísticas en los diarios de las localidades de procedencia hasta iniciativas de ayuda concreta.

\section{De la currícula a la extensión nuevamente}

Uno de los dispositivos que se estimuló y se apoyó desde la asignatura fue el planteo y presentación de un proyecto en el marco de la convocatoria de extensión 2017 de la UNLu. Una de las demandas de las que les estudiantes tomaron nota fue la necesidad de "romper el cerco mediático que no permite que la lucha tenga alcance a nivel país", tal como lo expresaron les asambleístas. Se elaboró una nueva propuesta de extensión de manera conjunta entre docentes, estudiantes, organizaciones sociales de Catamarca y de Buenos Aires, y la cátedra de la UNCa que participó en las actividades del viaje curricular.

Surgió así el proyecto que a la fecha sigue vigente, denominado: "Bienes comunes y universidad: coproducción de materiales de difusión para diferentes experiencias de lucha por la vida en la provincia de Catamarca" ${ }^{13}$ El mismo representa un enlace más entre la

12) Se prevén también actividades y formas de trabajo diferenciadas para estudiantes que por diferentes motivos no pudieron participar de la experiencia del viaje curricular. 13) RESHCS-LUJ:342/19. 
universidad y el territorio con el propósito de seguir estimulando procesos participativos de construcción de conocimientos para la transformación social, manteniendo una integralidad de las prácticas universitarias con las luchas por el territorio. Problematiza la función social de la universidad, su relación con la comunidad, en especial con empresas mineras, como Yacimientos Mineros Agua de Dionisio (YMAD), y busca estimular el diálogo de saberes y experiencias organizativas. Desde entonces las cohortes que realizaron el viaje curricular de la asignatura cada año trabajan de manera coordinada con les estudiantes que participan del proyecto de extensión. De este modo es posible enriquecer las actividades que contempla el viaje y potenciar las planificadas en el marco del proyecto. Cada nuevo viaje curricular ya no es solo un conjunto de acciones para la cohorte en cuestión, sino que también representa el momento de interacción y planificación para la realización de las propuestas planificadas en el proyecto.

El vínculo se retroalimenta de forma continua y permite el intercambio entre la universidad y el territorio. Se siguen trabajando y promoviendo el diálogo y los aprendizajes en formato de talleres in situ y, de acuerdo con cada necesidad, se proponen materiales de difusión. Se realizaron folletos y carteles para las cooperativas de trabajo agroecológico que fueron construidos y consensuados por estudiantes y cooperativistas con la finalidad de difundir los emprendimientos productivos y los beneficios de la agroecología. Se realizó un material audiovisual de difusión acerca de las consecuencias de la megaminería en los territorios y la lucha por los mismos. El video se elaboró mediante una relación continua de trabajo, desde el guion, el contenido, la edición, la presentación y la divulgación. El material no salió a la luz hasta que no contó con el visto bueno y la retroalimentación de todes les participantes, proceso que llevó tiempo pero que pudo concretarse recientemente. Los materiales de difusión se publicaron en redes sociales también por parte del equipo de trabajo. Se organizaron charlas virtuales en el marco de la pandemia, y debido a la imposibilidad de viajar, entre docentes, extensionistas y asambleístas para seguir trabajando y no perder las relaciones trazadas. En una de las charlas abiertas surgió la necesidad de elaborar a futuro, de manera conjunta con la cátedra de la UNCa y asambleístas, materiales que pongan en debate la aceptación de los fondos YMAD por parte de nuestras universidades.

\section{Algunas reflexiones parciales}

Para finalizar con la descripción de la experiencia, es necesario hacer algunos comentarios que no son menores. En primer lugar, comentar que la UNLu, en diciembre de 2018, cambió de idea respecto de los fondos YMAD, y mediante una iniciativa docente, en una sesión del Honorable Consejo Superior, con una Universidad prácticamente de vacaciones y a espaldas de la comunidad universitaria, decidió aceptarlos. El fatídico día 21 de diciembre, ante la presencia en el recinto de Nora Cortiñas, ${ }^{14}$ integrantes de la Asamblea El Algarrobo, el gremio docente y algunas agrupaciones estudiantiles que sostenían y defendían el rechazo de dichos fondos, la UNLu por resolución ${ }^{15}$ de dicho órgano consultivo tomó la inconsulta decisión de aceptarlos. La situación se volvió más difusa incluso cuando se leyó el articulado de dicha resolución, donde en su primer artículo reafirmaba el repudio a la

14) Integrante de Madres de Plaza de Mayo Línea Fundadora. Militante y defensora de los Derechos Humanos. 15) RESHCSLUJ:866/18. 
actividad extractiva a cielo abierto y sus consecuencias ambientales, sociales, económicas y políticas, pero en su segundo artículo aceptaba los fondos de dicha actividad.

Asimismo, es necesario mencionar que tanto en 2020 y 2021, por razones de público conocimiento a raíz de la pandemia de COVID-19, nos encontramos en actividades no presenciales en la Universidad, sosteniendo de manera remota las relacionadas con la docencia y en menor medida las que involucran extensión e investigación, por lo que muchas de las acciones planificadas se encuentran suspendidas y por lo tanto no es posible contar avances más recientes de la experiencia.

Además, al momento de la escritura de este artículo ${ }^{16}$ se encuentran privados de la libertad vecines y asambleístas de Andalgalá (Catamarca) luego de arbitrarios y violentos allanamientos a sus domicilios. Sucesos más que lamentables para dar cuenta de que el conflicto está activado más que nunca.

\section{Más humedales, menos inundaciones}

El segundo recorrido aborda las inundaciones ocurridas en el río Luján (Buenos Aires) y la necesidad de conservar los humedales para contener las mismas y minimizar sus efectos catastróficos a nivel urbano. La Figura 2 sintetiza el camino transitado.

Figura 2. Síntesis de la experiencia "Más Humedales, Menos Inundaciones”

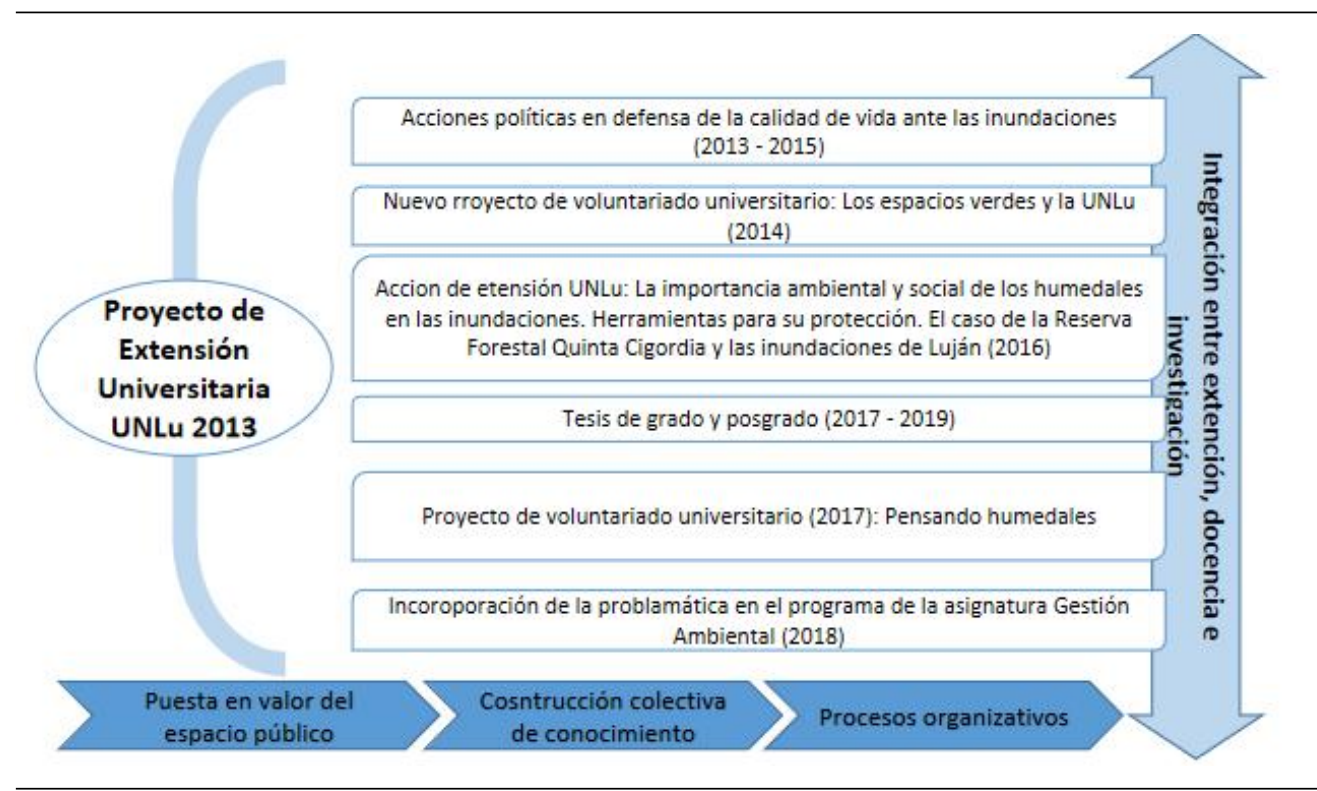

Fuente: Elaboración propia (2021)

\section{El contexto}

El partido de Luján está ubicado al noreste de la provincia de Buenos Aires, al oeste de la Región Metropolitana de Buenos Aires (RMBA tercera corona), y es uno de los municipios que integran la cuenca del río Luján. Dicho partido representa una superficie de $777,1 \mathrm{~km}^{2}$

16) 15 abril de 2021 . 
y es atravesado por el río del mismo nombre por su zona media en dirección NE, conforma una parte de la sección superior y la totalidad de la sección media de la cuenca. Se suman al curso del río los arroyos De las Acacias, El Chaña, Pereyra, Gutiérrez, El Haras y Lanusse.

Entre los años 2012 y 2015 se registraron nueve crecidas del río Luján de más de 3,80 metros, lo cual produjo su desborde e inundaciones de la ciudad homónima que requirieron la evacuación de aproximadamente 2000 personas en las zonas afectadas y causaron pérdidas materiales. Cuatro de las crecidas registradas han superado los 5,10 metros, inundaron el casco histórico y los barrios de la ciudad y causaron considerables pérdidas materiales a la población, las que, en la mayoría de los casos, fueron totales e incrementadas en cada nueva inundación.

La Reserva Forestal Quinta Cigordia es una ecozona ribera ubicada en la ciudad de Luján que se destaca por su densa población arbórea autóctona y exótica que genera el efecto de galería sobre los cursos de agua. Esta ecozona funciona como un espacio de resguardo de la biodiversidad, un paliativo a las tendencias actuales que vuelven uniformes las márgenes de aguas. Al mismo tiempo, la Quinta y su flora, con relación directa al río Luján, brindan un gran número de servicios ambientales que mitigan inundaciones y reducen los factores de contaminación urbanos.

Por su ubicación estratégica cercana al casco histórico de la ciudad, es un espacio favorable para la promoción de múltiples actividades recreativas y educativas. Sin embargo, el histórico abandono del espacio verde público por parte de organismos estatales ha disminuido la posibilidad de acceder a los servicios ambientales que brinda (recreación, usos múltiples del río, etc.). Esta problemática se ve agravada por el creciente y visible proceso de contaminación del arroyo Gutiérrez y del río Luján producto de múltiples elementos, como la industria, el agronegocio y/o los emprendimientos inmobiliarios. El abandono del espacio también dificulta la transitabilidad al interior de la Quinta debido al mal estado de los senderos, situación que fomenta la expulsión de la comunidad y el desaprovechamiento del espacio.

La Reserva Forestal Quinta Cigordia se encuentra ubicada en el Barrio Padre Varela, uno de los más afectados por las recientes y sucesivas inundaciones, situación que motiva la organización barrial y vecinal en el reclamo por mejorar las condiciones de vida de les vecines de la ciudad. A dicho proceso, a medida que las inundaciones eran más significativas en cuanto a su alcance y efectos, se fueron sumando otros barrios que aunaron reclamos.

Como equipo extensionista, militantes y vecines de la ciudad nos solidarizamos en la emergencia de cada inundación y acompañamos las acciones llevadas a adelante por la comunidad organizada, haciéndonos eco del reclamo por mejorar la calidad de vida, por minimizar el impacto de las inundaciones y cuidar el río, su costa y sus servicios ambientales.

En este contexto surgió el primer proyecto de extensión del equipo en esta temática, denominado "Los espacios verdes como enlace entre la UNLu y la ciudadanía de Luján". ${ }^{17}$ La propuesta nació de la relación y el trabajo político territorial entre el movimiento estudiantil y vecines de la ciudad de Luján. Contó con la aprobación y financiamiento de la UNLu, fue seleccionado en su Convocatoria de proyectos de extensión 2013, y forma parte del Programa de investigación Redes Epistémicas Ambientales, del Departamento de Ciencias Sociales. 
La meta fue promover la recuperación de la reserva forestal Quinta Cigordia como espacio de usos múltiples y ámbito de cogestión entre organismos públicos y la comunidad a partir de la generación de diversas iniciativas de participación que desde la perspectiva de la educación ambiental. Su finalidad fue impulsar la toma de conciencia sobre las problemáticas ambientales de la Quinta y sus inmediaciones y crear dinámicas colectivas de organización comunitaria dirigidas a la resolución y control de estas problemáticas.

Si bien la UNLu tiene un histórico anclaje en la producción de conocimiento en lo que respecta a la cuenca del río Luján y sus afluentes, entendemos que muchos de los materiales técnicos y científicos producidos en el ámbito académico son inaccesibles para la comunidad, lo cual dificulta el proceso de conocimiento y apropiación de ese espacio de carácter público. En este sentido, el proyecto no solo procuró recopilar y multiplicar la información científica generada en torno al conocimiento de esta reserva y de las problemáticas, sino también reconstruirla y redefinirla en el ámbito comunitario (Valverde, Poth, Moreno, 2018).

Igualmente, el proyecto se propuso construir, sobre la base de investigaciones ya existentes, encuestas de percepción ambiental y talleres y entrevistas colectivas, un mapeo histórico ambiental y un árbol de problemas ambientales que dieran cuenta de las condiciones actuales de la Quinta Cigordia y el arroyo Gutiérrez y de su evolución histórica. Además, buscó generar colectivamente materiales de difusión y cartelería.

Fueron reconocidas las problemáticas e identificadas las responsabilidades en cada caso, lo que permitió la construcción de un árbol de soluciones que facilitó la generación de estrategias de monitoreo, gestión, control y decisión comunitarias para el saneamiento, recuperación y manutención de este espacio público. También fortaleció los lazos con las instituciones públicas a partir de la elaboración de proyectos y planes de trabajo para concretar la efectiva categorización de la Quinta Cigordia como "Reserva natural urbana".

Esta primera experiencia en el territorio consolidó una dinámica de trabajo a partir de talleres y actividades vecinales de co-construcción de conocimiento con un carácter participativo, orientado a la acción pública. Se recuperó con vecines del barrio donde está la Quinta la historia ambiental de la misma a partir de sus protagonistas. Historia que la mayoría del equipo extensionista desconocía y que se vincula directamente con las inundaciones. Con el correr de los encuentros y los eventos catastróficos (2012-2015), se fue ampliando la red de relaciones políticas y solidarias con damnificades y organizaciones sociales de la cuenca, y es posible reconocer el espacio verde público en el que veníamos trabajando como parte del humedal. Este nuevo conocimiento incorporó la conformación de múltiples saberes y fortaleció el entramado relacional entre la Universidad y la comunidad.

El proyecto se desarrolló en un tiempo muy corto, ya que el escaso financiamiento no permitió continuar con las tareas de extensión. Por ello, en conjunto con la comunidad se elaboró una nueva propuesta de proyecto de voluntariado universitario en la convocatoria anual 2014 de la Secretaría de Políticas Públicas. En 2015 se puso en marcha el nuevo proyecto "Los espacios verdes y la UNLu". ${ }^{18}$ La visibilidad que alcanzó la reserva acercó a múltiples interesados e interesadas y las sucesivas inundaciones en el barrio fueron la base 
para la elaboración de un segundo tramo del proyecto inicial. Ese proyecto se propuso como metas generales:

- Fortalecer lazos comunitarios y solidarios al interior del barrio, por un lado, y con la Universidad, por el otro.

- Desarrollar herramientas teórico-prácticas asociadas al reconocimiento, control y gestión de las problemáticas ambientales a los fines de mitigar las condiciones de degradación ambiental y de vida de la zona.

- Promover la realización de espacios de formación y socialización en temas ligados a la cuenca del río Luján y sus afluentes: ecología, biología, derechos ambientales, producción y consumo sustentable de alimentos y todo lo referido a la educación ambiental.

La experiencia superó las expectativas iniciales en dos sentidos: en primer lugar, porque nos permitió considerar en conjunto (comunidad universitaria y vecines) a la reserva como parte de un humedal. Esto se dio en el marco de sucesivas inundaciones que tuvieron lugar mientras se desarrollaba el proyecto de extensión, cuando el barrio y la reserva fueron unos de los principales afectados por el agua y la contaminación de la misma. En este proceso se pudieron coordinar medidas coparticipativas no solo para la recuperación y revalorización del espacio público sino para el cuidado del humedal en el que está inmerso, a fin de minimizar los efectos de las crecidas del río y el arroyo. En segundo lugar, porque estimuló procesos de involucramiento de la sociedad y participación comunitaria al punto tal de abonar a la gestación de espacios de debate político, coordinación de acciones directas y difusión de actividades. Dichos espacios fueron conformados por vecines del barrio en el que se encuentra la Quinta Cigordia y llevan como nombre "Recuperemos la Quinta Cigordia e inundaciones nunca más".

El devenir de la experiencia y la necesidad de abordar el tema desde una mirada integral, aprendida con la comunidad, nos instó a seguir trabajando en el territorio, ahora en la defensa del humedal. Es así como en 2016, en el marco de la convocatoria UNLu 2015, nació la acción de extensión denominada "La importancia ambiental y social de los humedales en las inundaciones. Herramientas para su protección. El caso de la Reserva Forestal Quinta Cigordia y las inundaciones de Luján". ${ }^{19}$ Se dio continuidad a la planificación de talleres ambientales de la mano de vecines y los nuevos espacios organizativos antes mencionados. De esta manera se hizo difusión de la actual problemática de los humedales y las inundaciones a partir de materiales construidos colectivamente con la comunidad que daban cuenta de la necesidad de conservar los humedales para minimizar los impactos catastróficos de las inundaciones.

Este tipo de figuras (acción no articulada de extensión) en el contexto de las convocatorias de extensión de la UNLu tienen un alcance más acotado en cuanto a las actividades que se pueden realizar, los plazos y el financiamiento (es aun menor que el de los proyectos). Es por ello que se priorizó el encuentro con lacomunidad sobre la base de los diagnósticos realizados en las anteriores experiencias, se desarrollaron los talleres y se definieron estrategias participativas para el armado de materiales y su difusión en la ciudad.

19) Directora: Elda Tancredi. Codirectora: Alejandra Valverde. Aprobado por RESHCS-LUJ: 0000386-16, financiado por ESHCS-LUJ:0000612-16 y prorrogado por RESHCS-LUJ:471-18. 
Luego de un proceso de debate al interior del equipo y ante la necesidad de continuar con el trabajo, que fue interrumpido por la falta de financiamiento y los plazos administrativos, se elaboró de forma conjunta con un grupo de vecines una nueva propuesta de proyecto. En 2018 se inició el proyecto de voluntariado universitario presentado en la convocatoria 2017 "Compromiso Social Universitario de la Secretaría de Políticas Universitaria, denominado: Pensando humedales" ${ }^{20}$ Se dio continuidad al trabajo iniciado en la acción de extensión y se completaron los materiales de difusión elaborados en la acción anterior. Se participó en charlas abiertas a la comunidad donde les vecines difundieron los materiales y dieron charlas $y$ talleres en las instituciones educativas de la zona de influencia.

\section{De la extensión a la currícula}

De manera similar, y en paralelo con la experiencia de Catamarca, se fue incorporando la temática en la asignatura Gestión Ambiental para el abordaje de la problemática del uso y gestión pública de los humedales. El equipo docente de esta asignatura, del que formo parte, es el mismo que el de la asignatura Comercio Internacional y Ambiente, por lo que el proceso de debate para el tratamiento de la temática en la currícula se dio en los mismos términos. Al mismo tiempo, se pudieron incorporar pasantes estudiantiles de los proyectos de extensión anteriores y la temática es objeto de estudio para investigaciones de tesis de grado y posgrado.

La incorporación del conflicto socioambiental asociado a la conservación de humedales aún está en elaboración, ya que la primera experiencia propuesta fue planificada para el año 2020 y no fue posible avanzar porque las actividades universitarias se están desarrollando de manera virtual debido a la pandemia. Sea efectuaron en años anteriores algunas "pruebas piloto" de abordaje de la problemática que nos permitieron hacer diagnósticos y trazar una propuesta pedagógica. Nos queda todo un camino por delante para articular las experiencias del equipo de extensión con los contenidos de la asignatura y la recuperación del vínculo y las demandas del territorio.

\section{Algunas reflexiones parciales}

Entendemos que la experiencia no termina aquí, es fluctuante y depende de los pulsos y tiempos organizativos que se dan en los territorios. Nos encontramos en una situación muy particular a nivel mundial, la cual no nos permite por el momento encontrarnos en nuestros lugares de trabajo, estudio, militancia o vida social, por lo que muchas de las líneas sobre las que se pretende avanzar están interrumpidas. Hoy más que nunca esta situación sanitaria nos llama la atención acerca de las formas de producir y consumir, de nuestra alimentación y de la necesidad de preservar los humedales como barreas naturales para evitar futuras pandemias.

\section{Hacia la construcción de un modelo de universidad popular}

Es nuestro propósito como equipo de extensión ser parte de una universidad que responda a un modelo de formación crítica de profesionales, investigadores e investigadoras con compromiso en cuanto a la transformación social. De modo que la investigación esté dirigida a 
los "grandes problemas nacionales", a los sectores postergados y a los sujetos políticos colectivos. Una universidad donde la integralidad de las funciones sea una realidad y la extensión esté al mismo nivel que la enseñanza y la investigación (Tommasino, 2015). Este modelo apunta a una extensión crítica que, además de la integralidad de las funciones y el vínculo bidireccional, promueva formas de organización autónomas por parte de los sujetos sociales y políticos en conflicto como manera de construir respuestas a los problemas identificados. La integralidad de las prácticas universitarias para la extensión supone delinear algunas características que la constituyen, y la primera de ellas es considerar que la extensión es la puerta de entrada para la integración de las tareas docentes y de investigación y herramienta para la transformación de la sociedad. Por lo tanto, no puede darse de forma aislada o sin conectarla con las demás prácticas. Esto implica una forma diferente de concebir y practicar la enseñanza, la formación y la investigación.

El camino que elegimos es aquel que nos permita generar diferentes propuestas participativas que contribuyan, a partir de la práctica de les actores sociales involucrados, construir de modo colectivo políticas públicas que apunten al mejoramiento de la calidad de vida de la población. Es en ese sentido que la extensión universitaria se constituye en una herramienta colectiva y un desafío para el aporte de soluciones a los problemas que involucran la dimensión ambiental y se expresan territorialmente en forma de conflictos.

\section{Reflexiones finales}

Consideramos fundamental la práctica de la extensión en la formación de profesionales en la universidad pública para la construcción de conocimientos técnicos y humanos. Es por ello que no es posible pensarla descontextualizada ni deshistorizada. No solo porque aporta al proceso formativo en los conocimientos técnicos, sino porque fomenta el compromiso social y el espíritu de unidad frente a las relaciones de producción capitalistas.

La extensión como práctica integral permite al mismo tiempo la construcción de conocimiento colectivo. La vinculación con el territorio y el compromiso con las poblaciones con las que se trabaja posibilitan deconstruir conocimientos adquiridos en la universidad, recrearlos y reelaborarlos de manera conjunta para crear saberes. Así, no solo aportamos con nuestro bagaje de conocimientos sino que aprendemos otros que se constituyen en nuevos saberes.

Poder entender y practicar la extensión, la investigación y la docencia de modo integral ha llevado (y seguirá llevando) varios años de camino conjunto. En el mismo hay trabas burocráticas, estructurales y hasta de financiamiento por parte de la universidad. En la actualidad se están dando pasos importantes hacia la jerarquización de la extensión, pero aún falta bastante trabajo. Es necesario que las universidades públicas cuenten con financiamiento exclusivo para la extensión, como lo tienen para la docencia y la investigación, que dicho financiamiento sea duradero y que respete los tiempos de trabajo colectivo y no vaya detrás de los tiempos administrativos y burocráticos. Es preciso también que el trabajo de extensión pueda coordinarse con pasantías rentadas, becas estudiantiles, trabajos de investigación, de tesis, trabajos territoriales de los centros de estudiantes y las agrupaciones estudiantiles y docentes $\mathrm{y}$, fundamentalmente, que tenga un espacio en la currícula de las carreras. Se torna indispensable que les estudiantes de las distintas carreras participen de 
experiencias extensionistas y se comprometan con el trabajo y la formación en el territorio. Solo de esa manera creemos que se allanará un camino que, desde la Reforma Universitaria hasta este momento, apenas dio sus primeros pasos.

\section{Referencias bibliográficas}

Funtowicz, S. y Ravetz, J. (1993). Epistemología política. Ciencia con la gente. Centro Editor de América Latina. García, R. (2011). Interdisciplinariedad y sistemas complejos. Revista Latinoamericana de Metodología de las Ciencias Sociales, 1(1), 66-101. http://www.memoria.fahce.unlp.edu.ar/art_revistas/pr.4828/pr.4828.pdf

Merlinsky, G. (2014). Introducción. La cuestión ambiental en la agenda pública. En Merlinky (Comp.). Cartografías del conflicto ambiental en Argentina (pp. 19-60). CICCUS.

Proyecto institucional de la UNLu (2009-2012). RESAU-LUJ, 2-09 http://www.unlu.edu.ar/doc/proy-instest/0000002-09_anexo_pi.pdf

Tommasino, H. y Cano, A. (2015). Modelos de extensión universitaria en las universidades latinoamericanas en el siglo XXI: tendencias y controversias. Universidades, (67), 7-24. Unión de Universidades de América Latina y el Caribe Distrito Federal, Organismo Internacional. https://www.redalyc.org/pdf/373/37344015003.pdf

Valverde, A. (2020). Hacia la integralidad de las prácticas universitarias en el abordaje de los conflictos socioambientales desde una experiencia curricular. Red Sociales, Revista del Departamento de Ciencias Sociales, 07(06), 110-127.

Valverde, A.; Portugeis, A.; Lacko, E.; Bustos, L.; Bernardez, V. y Basualdo, S. (2019). Introducción. En Valverde, A. y Tancredi, E. (Comps.). Abordajes teórico-metodológicos de los conflictos socioambientales. Propuestas para un debate interdisciplinario a partir de estudios de caso. EdUNLU

Valverde, A.; Poth, C. y Moreno, L. (2018). ¿Por qué hacemos extensión en la universidad? Debates acerca de la integralidad de las prácticas a partir de dos experiencias de extensión universitaria. En Tancredi, E. (Dir.). Redes de saberes y decisiones políticas. Notaciones y anotaciones desde indagaciones compartidas. Grupo REDES.

Valverde, A.; Camarero, D.; Partucci, H.; Bojanich, L. y Ordoñez, S. (2015). Conflictos Socioambientales y Territoriales: propuestas teórico-metodológicas para su abordaje. XI Jornadas de Sociología. Facultad de Ciencias Sociales. Universidad de Buenos Aires.

Wagner, L. (2010). Problemas ambientales y conflicto social en Argentina. Movimientos socioambientales en Mendoza. La defensa del agua y el rechazo a la megaminería en los inicios del siglo XXI. Tesis doctoral Ciencias Sociales y Humanas. Universidad Nacional de Quilmes. 